Pacific

Journal of

Mathematics

CHEKANOV-ELIASHBERG INVARIANTS AND TRANSVERSE APPROXIMATIONS OF LEGENDRIAN KNOTS

Judith Epstein, Dmitry Fuchs, and Maike Meyer 


\title{
CHEKANOV-ELIASHBERG INVARIANTS AND TRANSVERSE APPROXIMATIONS OF LEGENDRIAN KNOTS
}

\author{
Judith Epstein, Dmitry Fuchs, And Maike Meyer
}

Topological isotopic, non-Legendrian isotopic, Legendrian knots in the standard contact space with equal ThurstonBennequin and Maslov numbers may have transverse isotopic transverse approximations.

\section{Introduction.}

In this article, we consider Legendrian and transverse knots in the standard contact space, that is in $\mathbb{R}^{3}$ with the contact structure globally given by the 1 -form $\alpha=y d x-d z$. It is well-known that a little push of an oriented Legendrian knot $\Gamma$ in the direction of its positive normal within the contact structure changes it into a transverse knot $\Gamma^{+}$, whose natural orientation, as determined by $\alpha$, matches the chosen orientation of $\Gamma$; pushing $\Gamma$ in the direction of its negative normal produces the transverse knot $\Gamma^{-}$of the opposite orientation.

Any transverse knot is transverse isotopic to $\Gamma^{+}$derived from some oriented Legendrian knot $\Gamma$, and if Legendrian knots $\Gamma, \Gamma^{\prime}$ are Legendrian isotopic, then $\Gamma^{+}, \Gamma^{\prime+}$ are transverse isotopic.

Besides their topological invariants, Legendrian and transverse knots have classical invariants of contact origin: The Thurston-Bennequin number $\tau \beta(\Gamma)$ and the Maslov number $\mu(\Gamma)$ for a Legendrian knot $\Gamma$ and the ThurstonBennequin number $\tau \beta(\Gamma)$ for a transverse knot $\Gamma$. Furthermore,

$$
\begin{aligned}
& \tau \beta\left(\Gamma^{+}\right)=\tau \beta(\Gamma)+\mu(\Gamma), \\
& \tau \beta\left(\Gamma^{-}\right)=\tau \beta(\Gamma)-\mu(\Gamma) .
\end{aligned}
$$

(For details see Bennequin's article $[\mathbf{B}]$.)

Question. If Legendrian knots $\Gamma, \Gamma^{\prime}$ have equal Thurston-Bennequin and Maslov invariants, and if $\Gamma^{+}$and $\Gamma^{\prime+}$ are transverse isotopic, then are $\Gamma$ and $\Gamma^{\prime}$ necessarily Legendrian isotopic?

It had not been possible to answer this question, at least negatively, before 1997, when new invariants of Legendrian knots were developed independently by Yu. Chekanov $[\mathbf{C}]$ and Ya. Eliashberg [E1, E2], Eliashberg's work was based on his earlier joint work with H. Hofer. Chekanov and 
Eliashberg each constructed examples of topologically isotopic Legendrian knots with equal Thurston-Bennequin and Maslov numbers, which are not Legendrian isotopic. In particular, Eliashberg [E1] considered a family of Legendrian knots $E(k, l)(k>0, l>0)$, which are given by their projections onto the $x z$-plane (see Figure 1 ); we refer to the Legendrian knots $E(k, l)$ as Eliashberg knots. Obviously, $E(k, l), E\left(k^{\prime}, l^{\prime}\right)$ are topologically isotopic if and only if $k+l=k^{\prime}+l^{\prime}$. An easy computation shows that $\mu(E(k, l))=0$, independently of $k, l$, and that

$$
\tau \beta(E(k, l))= \begin{cases}1, & \text { if } k+l \text { is even } \\ -3, & \text { if } k+l \text { is odd }\end{cases}
$$

Eliashberg proved that $E(1,3)$ and $E(2,2)$ are not Legendrian isotopic.

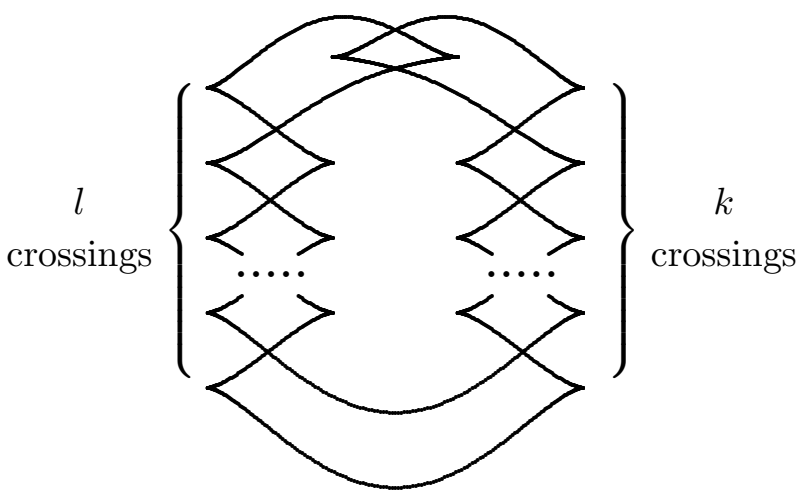

Figure 1. $E(k, l)$.

Our results concern both Legendrian and transverse representations of Eliashberg knots. First, we demonstrate that $E(k, l)^{ \pm}$are not all transversely different.

Theorem 2.2. If $l$ is even, then $E(k, l)^{+}$and $E(k-1, l+1)^{+}$are transverse isotopic; if $l$ is odd, then $E(k, l)^{-}$and $E(k-1, l+1)^{-}$are transverse isotopic.

Then, using the graded version of the linearization of Chekanov's invariant, we prove the following result.

Theorem 4.1. The Legendrian knots $E(k, l)$ and $E\left(k^{\prime}, l^{\prime}\right)$ are Legendrian isotopic if and only if $k=k^{\prime}, l=l^{\prime}$ or $k=l^{\prime}, l=k^{\prime}$.

This result is also mentioned in $[\mathbf{C}]$, but some details of the computation of Chekanov's invariant are germane to our proof, and we present them in Section 4. Together, these theorems answer the question above in the negative. 
Conjecture. If $l$ is odd, then $E(k, l)^{+}$and $E(k-1, l+1)^{+}$are not transverse isotopic; if $l$ is even, then $E(k, l)^{-}$and $E(k-1, l+1)^{-}$are not transverse isotopic.

This conjecture, motivated solely by our inability to construct transverse isotopies for these parities of $l$, implies that topologically isotopic transverse knots with equal Thurston-Bennequin numbers are not necessarily transverse isotopic.

We are grateful to Yura Chekanov and Yasha Eliashberg for their beautiful breakthrough in contact topology. We also want to thank Yasha for his generous and patient explanations of his published and unpublished results.

\section{Eliashberg knots and transverse isotopies.}

We recall that the projection of a generic Legendrian curve onto the $x z$ plane is a smooth curve without vertical tangents and with no singularities other than isolated cusps and transverse double crossings; this projection completely determines the Legendrian curve. Closed curves as above are projections of Legendrian knots, and we call them $x z$-diagrams. Two Legendrian knots are Legendrian isotopic if and only if the $x z$-diagram of the first may be transformed into the $x z$-diagram of the second through admissible moves of the three kinds shown in Figure 2.

I.

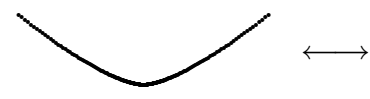

II.
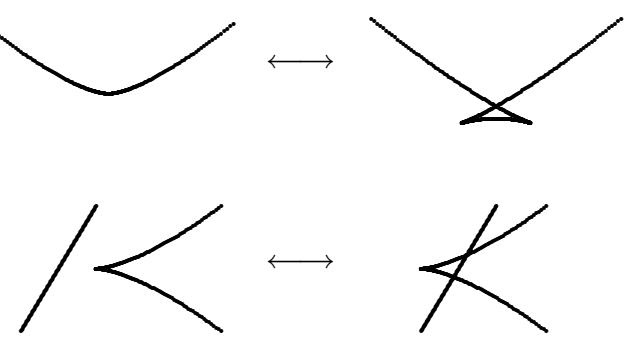

III.





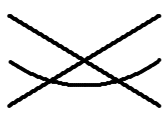

Figure 2.

There exist direct ways of computing the Thurston-Bennequin and Maslov numbers of a Legendrian knot given by an $x z$-diagram. In order to compute $\tau \beta(\Gamma)$, we must orient $\Gamma$, although the final result does not depend on our choice of orientation. If we count the number $l$ of left cusps, the number $c_{1}$ of same-direction crossings $\chi$ or $\mathbb{X}$, and the number $c_{2}$ of opposite-direction crossings $X$ or $\chi$ in the $x z$-diagram of $\Gamma$, then

$$
\tau \beta(\Gamma)=c_{1}-c_{2}-l .
$$


The Maslov number of an oriented Legendrian knot $\Gamma$ is determined by the formula

$$
\mu(\Gamma)=\frac{1}{2}(d-u)
$$

where $d$ is the number of downward cusps and $u$ is the number of upward cusps in the $x z$-diagram of $\Gamma$ (see Figure 3). The Maslov number does depend on the choice of orientation. If $-\Gamma$ denotes $\Gamma$ with the opposite orientation, then $\mu(-\Gamma)=-\mu(\Gamma)$.

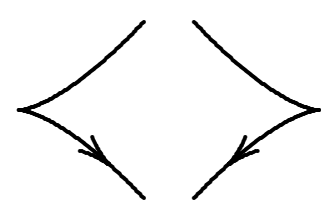

downward

cusps

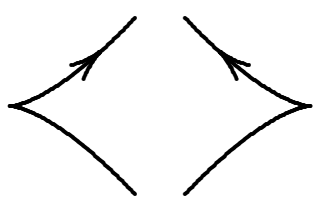

upward

cusps

Figure 3.

Applying this procedure to Figure 1, we easily get the result stated in the introduction:

$$
\begin{aligned}
\tau \beta(E(k, l)) & = \begin{cases}1, & \text { if } k+l \text { is even } \\
-3, & \text { if } k+l \text { is odd }\end{cases} \\
\mu(E(k, l)) & =0 .
\end{aligned}
$$

The $x z$-projection of a smooth transverse curve is always a smooth oriented curve. The transversality condition $(y d x-d z>0)$ means that at a point where the diagram is oriented to the right $(d x>0)$, the $y$-coordinate is greater than the slope $(d z / d x)$, and at a point where the diagram is oriented to the left $(d x<0)$, the $y$-coordinate is less than the slope. In particular, if, at a transverse crossing point of the $x z$-projection of a transverse curve, the upward direction lies between two positive half-strands $(d z>0)$, then the $y$-coordinate of the strand directed left is greater than the $y$-coordinate of the strand directed right. Also, all vertical tangent vectors $(d x=0)$ are directed downward $(d z<0)$.

We will present transverse knots by generic $x z$-diagrams, that is closed smooth oriented curves with only double transverse crossings of non-vertical strands and with no inflection points having vertical tangents. In space with a right-handed orientation, if the $x$-axis is directed right and the $z$ axis is directed up, then the $y$-axis will be directed away from the observer. Therefore, at a crossing, the strand with the greater value of the $y$-coordinate should be drawn as the lower strand. The properties of $x z$-projections of transverse curves described above show that the configurations shown in Figure 4 are never possible for generic $x z$-diagrams of transverse knots. On 
the other hand, a generic $x z$-diagram without the configurations of Figure 4 always determines a transverse isotopy class of a transverse knot.

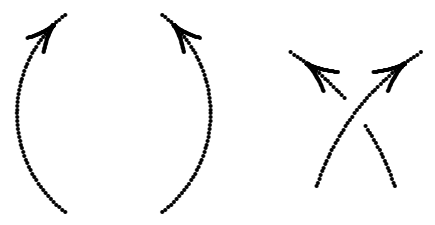

Figure 4.

The transition from a generic $x z$-diagram of an oriented Legendrian knot $\Gamma$ to a generic $x z$-diagram of the transverse $k$ not $\Gamma^{+}$is brought about by the modifications shown in Figure 5.
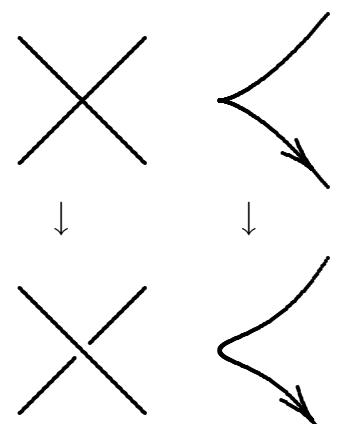
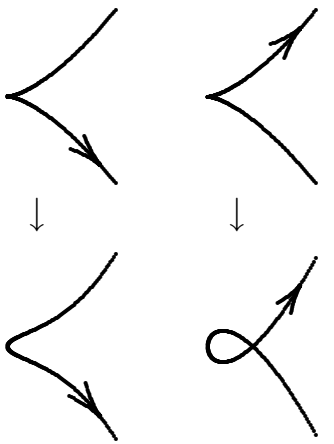

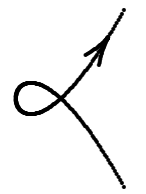



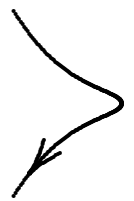

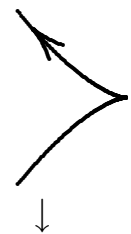

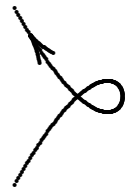

Figure 5 .

In general, there are many ways to construct an oriented Legendrian knot $\Gamma$ from a transverse knot, whose $x z$-diagram $\Delta$ represents the transverse isotopy class of $\Gamma^{+}$. Those depicted in Figure 6, when followed by the modifications in Figure 5, preserve the transverse isotopy type of $\Delta$.
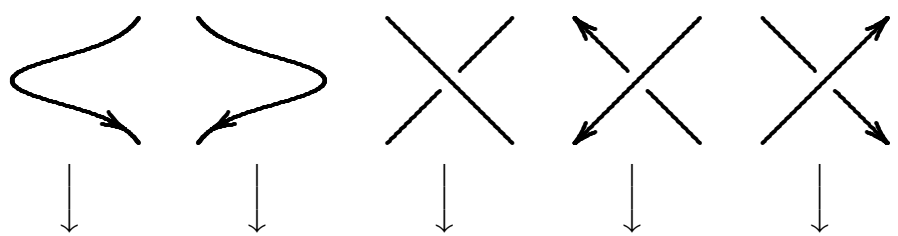

$\downarrow \downarrow$
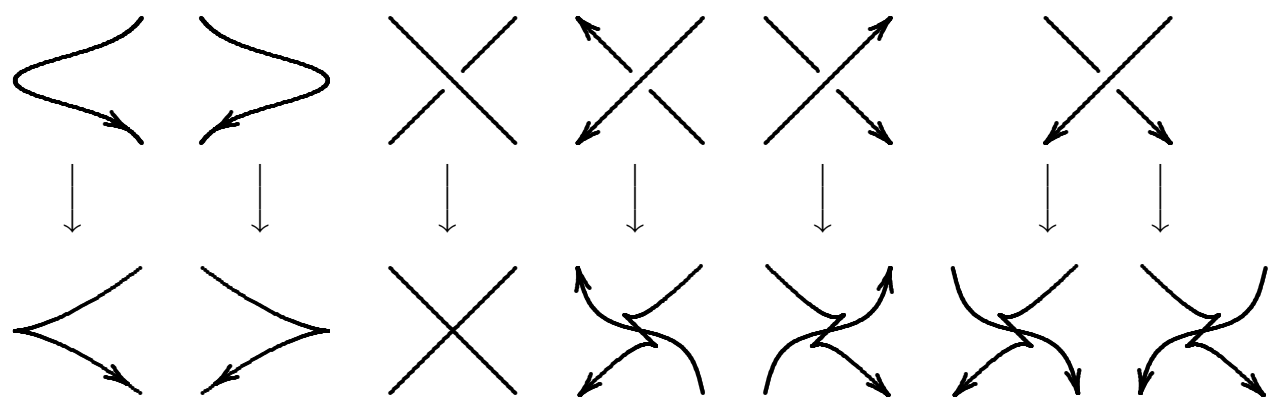

Figure 6. 
As in the paper of one of the authors and Serge Tabachnikov $[\mathbf{F T}]$, we will apply two standard modifications to the $x z$-diagrams of oriented Legendrian knots within a topological isotopy class, which change their Legendrian isotopy type. Namely, adding a zig means creating two new upward cusps without cusps or crossings between them and adding a zag is doing the same with downward cusps (see Figure 7). Inverse operations are removing zigs or removing zags.
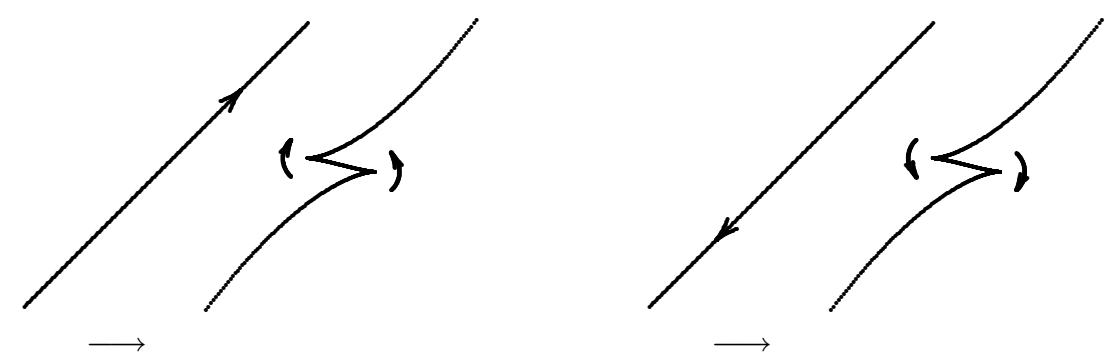

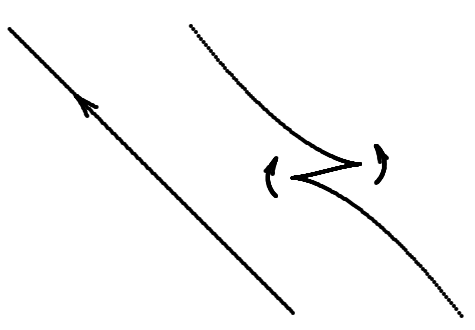

adding a zig

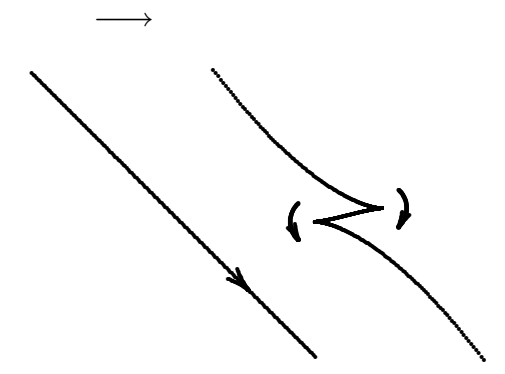

adding a zag

Figure 7.

We note that adding a zig or a zag decreases the Thurston-Bennequin number by 1; adding a zig decreases the Maslov number by 1 , and adding a zag increases the Maslov number by 1 . Hence, adding a zig to $\Gamma$ does not affect the Thurston-Bennequin number of $\Gamma^{-}$, and adding a zag to $\Gamma$ does not affect the Thurston-Bennequin number of $\Gamma^{+}$.

In $[\mathbf{F T}]$, the authors prove that two topologically isotopic oriented Legendrian knots become Legendrian isotopic after adding sufficiently many zigs and/or zags to each of them (in the terminology of $[\mathbf{F T}]$, sufficiently many zigzags). The theorem below seems to be known to many people in contact topology, but we were not able to trace it in the literature, and we give its proof (which is similar to the proof of the above-mentioned result in $[\mathbf{F T}]$ ). For the sake of convenience, we introduce the following terminology.

We call two oriented Legendrian knots zig-isotopic, if they become Legendrian isotopic after adding sufficiently many zigs to each of them; thus, a zig-isotopy is a transformation of an oriented Legendrian diagram by the 
standard moves of Figure 2, along with the addition and removal of zigs. A zag-isotopy is defined in a similar way.

Theorem 2.1. Let $\Gamma, \Gamma^{\prime}$ be oriented Legendrian knots. The transverse knots $\Gamma^{+}, \Gamma^{+}$are transverse isotopic if and only if $\Gamma, \Gamma^{\prime}$ are zag-isotopic. Similarly, $\Gamma^{-}, \Gamma^{-}$are transverse isotopic if and only if $\Gamma, \Gamma^{\prime}$ are zig-isotopic.

Proof. The zig and zag parts of Theorem are completely similar, so we restrict our attention to zags. We need to prove two things:

(i) The addition of a zag to $\Gamma$ does not change the transverse isotopic type of $\Gamma^{+}$.

(ii) If $\Gamma^{+}, \Gamma^{\prime+}$ are transverse isotopic, then $\Gamma, \Gamma^{\prime}$ are zag-isotopic.

Part (i) is simple. Adding a zag to $\Gamma$ results in a modification of the diagram of $\Gamma^{+}$, which is removable by a transverse isotopy (see Figure 8).
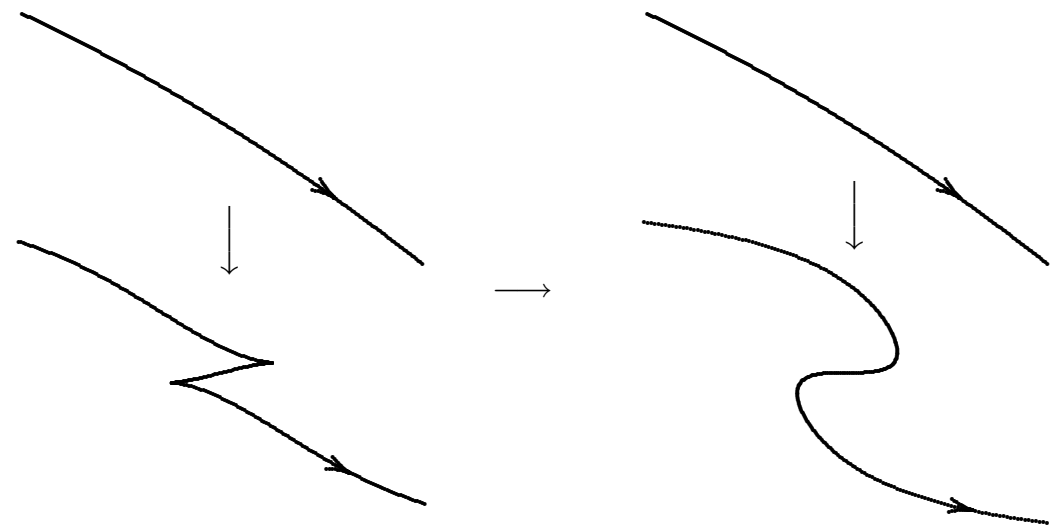

Figure 8.

To prove part (ii), we consider oriented Legendrian knots $\Gamma, \Gamma^{\prime}$ presented by generic $x z$-diagrams. Generic $x z$-diagrams of $\Gamma^{+}, \Gamma^{++}$can be obtained from those of $\Gamma, \Gamma^{\prime}$ by the modifications shown in Figure 5 . Since $\Gamma^{+}, \Gamma^{\prime+}$ are transverse isotopic, then the diagram of $\Gamma^{+}$can be smoothly deformed into the diagram of $\Gamma^{\prime+}$ with finitely many violations of genericity, as defined above; namely, for finitely many values of the parameter, the diagram will have one of the following four kinds of singularities:

A. A triple point (with the three crossing strands being transverse and non-vertical).

B. A point of first-order self-tangency (of non-vertical strands).

C. A (non-crossing) inflection point with a vertical tangent.

D. A crossing of a vertical and a non-vertical strand (with no inflection point on the vertical strand). 
Let $\Gamma^{+}=\Delta_{0}, \Delta_{1}, \ldots, \Delta_{n}=\Gamma^{\prime+}$ be a sequence of generic diagrams from the deformation with precisely one non-removable singularity of the types AD between $\Delta_{i-1}, \Delta_{i}(i=1, \ldots, n)$. Let $\Gamma_{0}=\Gamma, \Gamma_{n}=\Gamma^{\prime}$, and let $\Gamma_{1}, \ldots, \Gamma_{n-1}$ be obtained from $\Delta_{1}, \ldots, \Delta_{n-1}$ as shown in Figure 6 . We need to prove that $\Gamma_{i-1}, \Gamma_{i}$ are zag-isotopic for each $i$.

First, we remark that the indeterminacy of the constructions in Figure 6 does not really matter. Legendrian $x z$-diagrams obtained from the same transverse $x z$-diagram by different modifications allowed by Figure 6 are zag-isotopic; this is proved in Figure 9. (Here and on, we assume that the isotopy is constant outside a neighborhood of the singularity.)

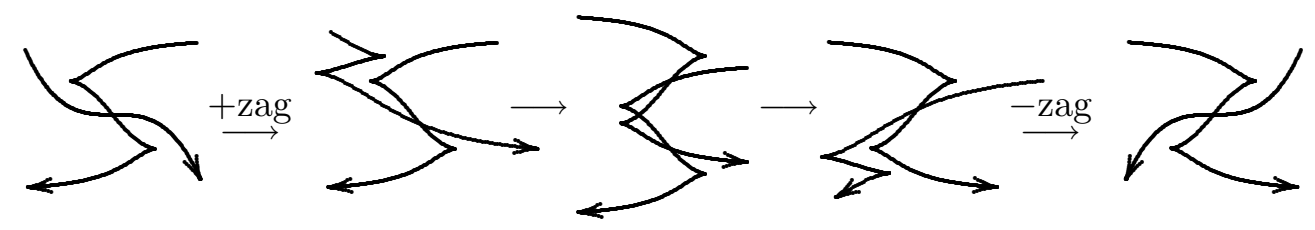

Figure 9.

Thus, we may take generic transverse $x z$-diagrams $\Delta, \Delta^{\prime}$, such that $\Delta$ can be smoothly deformed into $\Delta^{\prime}$ with one non-removable singularity of the types A-D and convert $\Delta, \Delta^{\prime}$ into generic $x z$-diagrams $\Gamma, \Gamma^{\prime}$ by the modifications allowed by Figure 6 . If the latter is not unique, we can choose one that is more convenient to us. Finally, we prove that $\Gamma, \Gamma^{\prime}$ are zag-isotopic. We will have to consider each of the cases of the types A-D separately; these cases will be split into subcases corresponding to the different direction of strands, etc.

Case A. This case requires the greatest amount of work. We order the three strands of the triple point by decreasing slope. Let $y_{i}$ be the value of the $y$-coordinate at the crossing point of the $i$-th strand. We introduce the permutation $\left(i_{1}, i_{2}, i_{3}\right)$ by the condition $y_{i_{1}}<y_{i_{2}}<y_{i_{3}}$ and set $d_{i}=1$, if the $i$-th strand is directed right and $d_{i}=-1$, if it is directed left. The permutation $\left(i_{1}, i_{2}, i_{3}\right)$ and the numbers $d_{1}, d_{2}, d_{3}$ specify the 48 subcases of Case A, 14 of which are prohibited by the following condition derived from Figure 4:

$$
\text { It is impossible that } s<t, i_{s}<i_{t}, d_{s}=1, d_{t}=-1 \text {. }
$$

Fortunately, we can avoid considering the remaining 34 cases separately. We note that at the intersection of strands labelled as $s$-th and $t$-th with $s<t$ the Legendrian diagram acquires a pair of cusps if and only if $i_{s}<i_{t}$ (see Figure 6). 
If $i_{3}=3$ and $d_{3}=1$, then no matter what $i_{1}, i_{2}, d_{1}, d_{2}$ are, a zag-isotopy between $\Gamma$ and $\Gamma^{\prime}$ may be performed as shown in Figure 10. The case of $i_{1}=1$ and $d_{1}=-1$ is treated in a similar way.
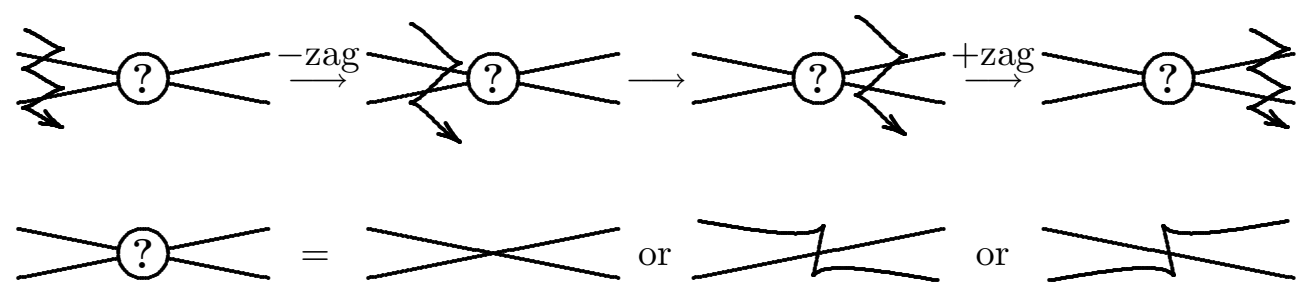

Figure 10.

If $i_{3}=3$ and $d_{3}=-1$, then the condition $(*)$ implies that $d_{1}=d_{2}=-1$. Since the case $i_{1}=1$ has been already considered, we may set $i_{1}=2$ and $i_{2}=1$. In this case, $\Gamma$ and $\Gamma^{\prime}$ are Legendrian isotopic, as shown in Figure 11, and thus zag-isotopic. The case of $i_{1}=1$ and $d_{3}=1$ is treated in a similar way.
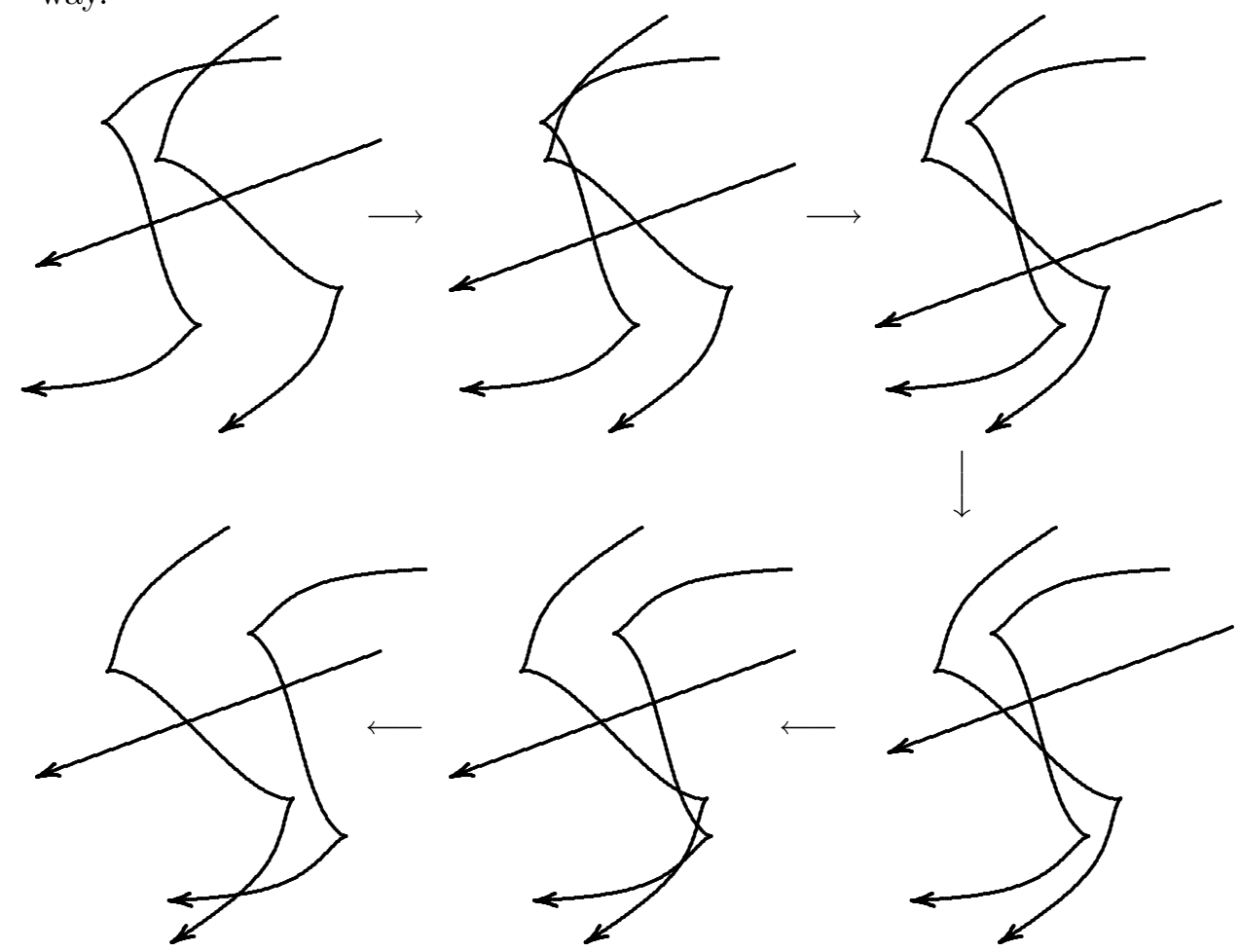

Figure 11.

It remains to consider the cases for which $i_{1} \neq 1$ and $i_{3} \neq 3$. These are the cases in which $\left(i_{1}, i_{2}, i_{3}\right)=(2,3,1),(3,1,2)$, or $(3,2,1)$. For the first two 
permutations, the corresponding Legendrian diagram has only one pair of cusps, and the Legendrian isotopy is obvious (see Figure 12 for an example). In the final case, there are no cusps at all, and the Legendrian isotopy is simply the third move of Figure 2.


Figure 12.

Case B. On transverse diagrams, this case corresponds to the birth or the death of two crossings. Depending on the directions of the strands and the orderings of $y$-coordinates, there are 8 possible subcases, 2 of which are prohibited by the second condition of Figure 4 . In the remaining 6 subcases, the zag-isotopy between the two Legendrian diagrams is the same up to reflections in the $x$ - and $z$-axes: Simply, a zag is created on one strand, after which one of the cusps passes through the other strand (see Figure 13 for an example).
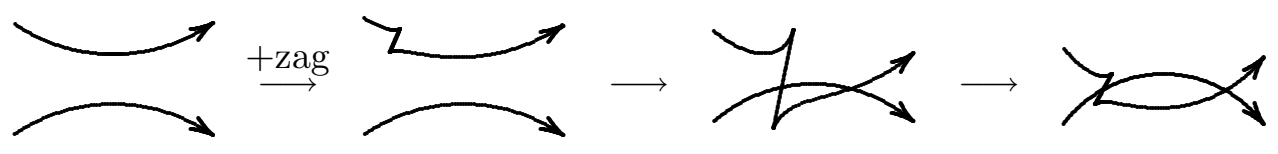

Figure 13.

Case C. This case is especially easy, since it involves only one strand. The birth or the death of a pair of points with vertical tangents directed downward obviously corresponds to the addition or deletion of a zag (see Figure 8).
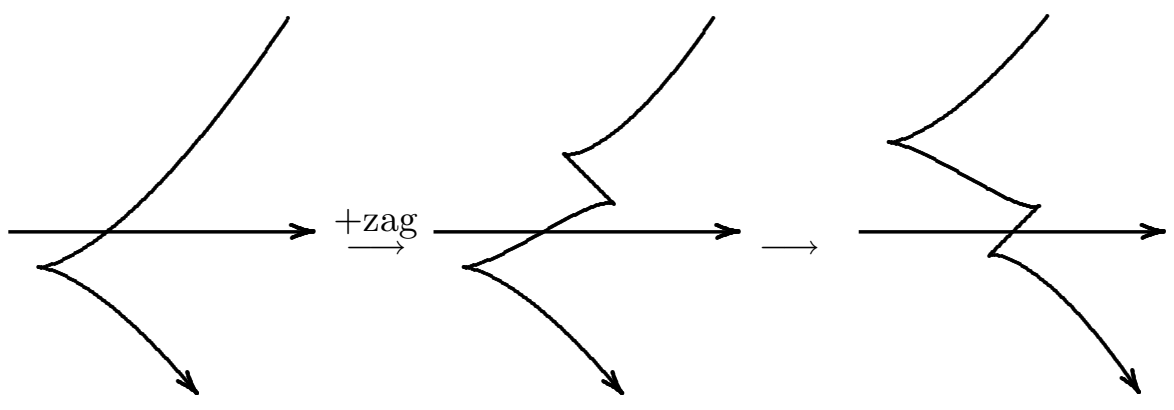

Figure 14. 
Case D. On a transverse diagram, this case corresponds to the passing of a point with a downward vertical tangent through a non-vertical strand. There are 8 subcases, depending on the direction of concavity of the vertical strand, the direction of the non-vertical strand, and the order of the $y$-coordinates. None are prohibited by the conditions of Figure 4 . The zag-isotopies between the Legendrian diagrams in all the subcases are very similar; an example is shown in Figure 14.

Theorem 2.2. Let $E(k, l)$ be the oriented Legendrian knot depicted in the introduction (see Figure 1). If $l$ is even, then the transverse knots $E(k, l)^{+}$, $E(k+1, l-1)^{+}$are transverse isotopic. If $l$ is odd, then the transverse knots $E(k, l)^{-}, E(k+1, l-1)^{-}$are transverse isotopic.

Proof. Depending on the parity of $l$, we need to construct either a zagisotopy or a zig-isotopy between $E(k, l)$ and $E(k-1, l+1)$. The case of even $l$ is shown in Figure 15; the case of odd $l$ is similar.

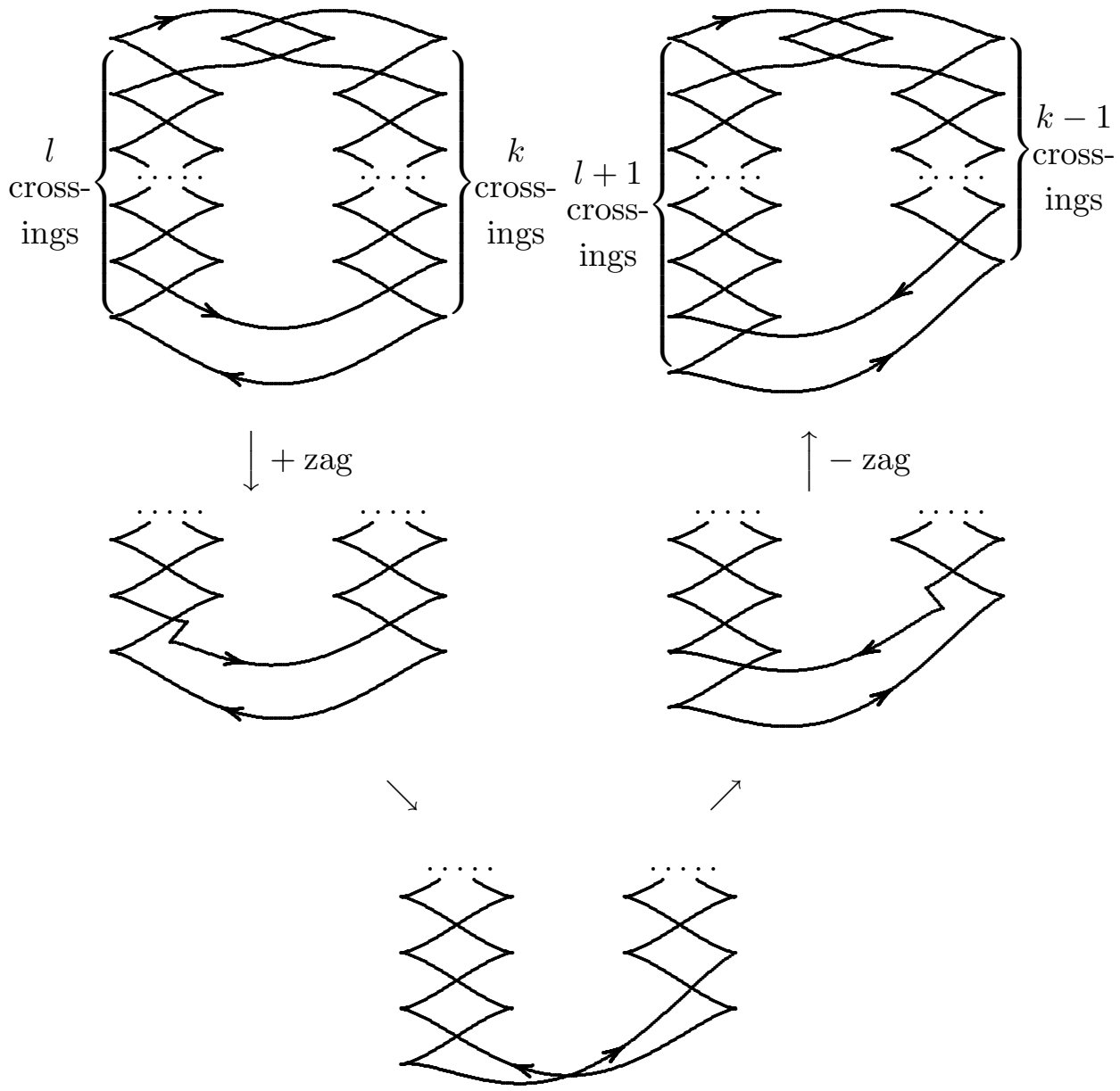

Figure 15. 


\section{Chekanov-Eliashberg invariants.}

Let $\Gamma$ be an oriented Legendrian knot represented by a generic $x y$-diagram. At any crossing $\mathbf{x}$, the two strands form four angles, of which two are deemed positive and two negative. The positive crossings of $\mathbf{x}$ are bounded on the right by the upper strand and on the left by the lower strand (see Figure 16).

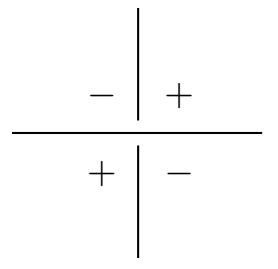

Figure 16.

For $n \geq 0$, we fix a convex planar domain $P_{n}$, bounded by a piecewise smooth curve $\Pi_{n}$ of $n+1$ vertices, which are denoted in a counterclockwise direction as $u_{0}, u_{1}, \ldots, u_{n}$; when $n \geq 2, P_{n}$ may be a regular $(n+1)$-gon. We now consider orientation preserving immersions $f$ of $P_{n}$ into the $x y$-plane, which map $\Pi_{n}$ into the diagram of $\Gamma$ in such a way that each $u_{i}$ is mapped to a crossing. Thus, $f\left(\Pi_{n}\right)$ changes strands at $f\left(u_{i}\right)$. Two such immersions are isotopic, if they can be connected by a regular isotopy within the class of immersions defined above.

We restrict our attention to only those immersions, whose images of vertices form a single positive angle at $f\left(u_{0}\right)$. For any $n$, the set of isotopy classes of such immersions $f$ with $f\left(u_{0}\right)=\mathbf{x}$ is denoted as $\operatorname{Imm}_{n}(\mathbf{x})$. We define the function $h$ on each crossing of $\Gamma$ to be the absolute value of the difference of the $z$-coordinates of the points above the crossing.

Proposition $3.1([\mathbf{C}])$. For any $f \in \operatorname{Imm}_{n}(\mathbf{x})$,

$$
h(\mathbf{x})-\sum_{i=1}^{n} h\left(f\left(u_{i}\right)\right)>0 .
$$

Proof. Since $\Gamma$ is Legendrian, the difference on the left hand side of the inequality is equal to the area of the image of $f$, that is, to $\int_{P_{n}} f^{*}(d x \wedge d y)$, which is positive.

Corollary $3.2([\mathbf{C}])$. For any $\mathbf{x}, \bigcup_{n=0}^{\infty} \operatorname{Imm}_{n}(\mathbf{x})$ is finite.

Let $\mathbf{A}$ denote the differential $\mathbb{Z}_{2}$-algebra, which is defined as the free associative algebra generated by the crossings of the $x y$-diagram of $\Gamma$, whose differential $d: \mathbf{A} \rightarrow \mathbf{A}$ satisfies the product rule and is given by the formula

$$
d(\mathbf{x})=\sum_{n=0}^{\infty} \sum_{f \in \operatorname{Imm}_{n}(\mathbf{x})} f\left(u_{1}\right) \ldots f\left(u_{n}\right) .
$$


Technically, the following two theorems constitute the main results of Chekanov and Eliashberg.

Theorem 3.3 ([C, E1]). For $d$ as above, $d^{2}=0$.

Theorem $3.4([\mathbf{C}, \mathbf{E} 1])$. Let $\mathbf{H}=\operatorname{Ker} d / \operatorname{Im} d$, then $\operatorname{dim} \mathbf{H}$ is a Legendrian isotopy invariant of $\Gamma$.

Chekanov's paper $[\mathbf{C}]$ contains a potentially stronger result. For a differential $\mathbb{Z}_{2}$-algebra $\mathbf{B}$, let $\mathbf{S} B$ denote the differential $\mathbb{Z}_{2}$-algebra, which is obtained from $\mathbf{B}$ by adding two free generators $\mathbf{a}$ and $\mathbf{b}$ and by extending the differential $d$ by $d(\mathbf{a})=\mathbf{b}$ and $d(\mathbf{b})=0$. We say that $\mathbf{B}$ and $\mathbf{B}^{\prime}$ are stably isomorphic, if $\mathbf{S}^{n} \mathbf{B}$ is isomorphic as a differential $\mathbb{Z}_{2}$-algebra to $\mathbf{S}^{m} \mathbf{B}^{\prime}$ for some $n, m$.

Theorem $3.5([\mathbf{C}])$. The stable isomorphism type of $\mathbf{A}$ is a Legendrian isotopy invariant of $\Gamma$.

Obviously, Theorem 3.5 implies Theorem 3.4.

It is not known whether the invariants described in Theorems 3.4 and 3.5 are actually new invariants of Legendrian isotopy classes. However, Chekanov's and Eliashberg's invariant has a graded version, which is known to distinguish Legendrian isotopy classes not distinguished by earlier invariants. This graded version is especially useful when the Maslov number $\mu$ of $\Gamma$ is 0 .

In order to introduce a grading, we need to fix an orientation of $\Gamma$, although the degrees $\operatorname{deg} \mathbf{x}$, defined below, do not depend on this orientation. For a point $x \in \Gamma$, let $\Phi(x)$ denote the angle (measured counterclockwise) from the positive direction of the $-x$-axis to the $x y$-projection of the positive tangent to $\Gamma$ at $x$. The function $\Phi$ is multivalued; $\Phi(x)$ is defined up to the addition of integral multiples of $2 \pi$. If $\mu=0$, then $\Phi$ has a continuous branch $\varphi: \Gamma \rightarrow \mathbb{R}$. If $\mu \neq 0$, then $\Phi$ has a continuous branch $\varphi: \Gamma \rightarrow \mathbb{R} / 2 \pi|\mu| \mathbb{Z}$.

Let $\mathbf{x}$ be a crossing of the $x y$-diagram of $\Gamma$ and $x_{1}, x_{2} \in \Gamma$ be the two points above $\mathbf{x}$. Let $\alpha(\mathbf{x})$ be the measure of the positive angle at $\mathbf{x}$ (thus, $0<\alpha(\mathbf{x})<\pi)$. We also suppose that $x_{1}$ lies below $x_{2}$, that is, the $z$ coordinate of $x_{2}$ is less than that of $x_{1}$.

Proposition 3.6. The number

$$
\varphi\left(x_{1}\right)-\varphi\left(x_{2}\right)-\alpha(\mathbf{x})
$$

is an integral multiple of $\pi$.

Proof. The difference $\varphi\left(x_{1}\right)-\varphi\left(x_{2}\right)$ is congruent modulo $2 \pi$ to the counterclockwise angle from the positive direction of the upper strand at $\mathbf{x}$ to the positive direction of the lower strand at $\mathbf{x}$. As seen in Figure 16, this angle is equal either to the positive angle or to twice the positive angle plus the negative angle. In other words, this angle is either $\alpha(\mathbf{x})$ or 
$2 \alpha(\mathbf{x})+\pi-\alpha(\mathbf{x})=\alpha(\mathbf{x})+\pi$. Hence, $\varphi\left(x_{1}\right)-\varphi\left(x_{2}\right)-\alpha(\mathbf{x})$ is congruent mod $2 \pi$ to either 0 or $\pi$.

\section{Definition.}

$$
\operatorname{deg}(\mathbf{x})=\frac{1}{\pi}\left(\varphi\left(x_{1}\right)-\varphi\left(x_{2}\right)-\alpha(\mathbf{x})\right)
$$

Obviously,

$$
\operatorname{deg}(\mathbf{x}) \in \begin{cases}\mathbb{Z}, & \text { if } \mu=0, \\ \mathbb{Z}_{2|\mu|}, & \text { if } \mu \neq 0 .\end{cases}
$$

Using this definition, we introduce a $\mathbb{Z}$ - or a $\mathbb{Z}_{2|\mu|}$-grading in $\mathbf{A}$ :

$$
\operatorname{deg}\left(\mathbf{x}_{1} \ldots \mathbf{x}_{n}\right)=\operatorname{deg}\left(\mathbf{x}_{1}\right)+\cdots+\operatorname{deg}\left(\mathbf{x}_{n}\right) .
$$

Proposition 3.7. The differential $d: \mathbf{A} \rightarrow \mathbf{A}$ is homogeneous of degree -1 .

For a proof, see [C], Lemma 7.4.

Proposition 3.7 shows that the cohomology $\mathbf{H}$ is graded:

$$
\mathbf{H}=\bigoplus_{r \in \mathbb{Z}} \mathbf{H}_{r} \quad \text { or } \quad \mathbf{H}=\bigoplus_{r \in \mathbb{Z} / 2|\mu| \mathbb{Z}} \mathbf{H}_{r} .
$$

The following theorem is an extension of Theorem 3.4.

Theorem 3.8 ([C, E1]). The dimensions $\operatorname{dim} \mathbf{H}_{r}$ are Legendrian isotopy invariants of $\Gamma$.

Remark. Usually, we organize these dimensions into the Poincaré series or polynomial:

$$
\operatorname{Poi}(\mathbf{H})=\sum_{r \in \mathbb{Z}}\left(\operatorname{dim} \mathbf{H}_{r}\right) t^{r} \text { or } \sum_{r \in \mathbb{Z} / 2|\mu| \mathbb{Z}}\left(\operatorname{dim} \mathbf{H}_{r}\right) t^{r}
$$

(in the second case, we assume that $t^{2|\mu|}=1$ ).

Theorem 3.5 also has a graded version, which we omit here.

We will also need a coarser invariant, developed by Chekanov.

Definition. An algebra homomorphism $\varepsilon: \mathbf{A} \rightarrow \mathbb{Z}_{2}$ is called an augmentation, if $\varepsilon \circ d=0$. An augmentation $\varepsilon$ is called graded, if $\varepsilon(a)=0$ for any homogeneous $a \in \mathbf{A}$, such that $\operatorname{deg} a \neq 0$.

It should be noted that augmentations do not exist for all Legendrian diagrams.

Let $\varepsilon: \mathbf{A} \rightarrow \mathbb{Z}_{2}$ be an augmentation. Let $\mathbf{A}_{0}=\operatorname{Ker} \varepsilon$ and $\mathbf{A}_{0}^{k}=$ $\underbrace{\mathbf{A}_{0} \cdots \mathbf{A}_{0}}_{k}$. Since $d(a) \in \operatorname{Ker} \varepsilon=\mathbf{A}_{0}$ for $a \in \mathbf{A}$, then

$$
d\left(a_{1} \ldots a_{k}\right)=\sum_{i=1}^{k} a_{1} \ldots a_{i-1} d\left(a_{i}\right) a_{i+1} \ldots a_{k} \in \mathbf{A}_{0}^{k}
$$


for any $a_{1}, \ldots, a_{k} \in \mathbf{A}_{0}$. Thus,

$$
d\left(\mathbf{A}_{0}^{k}\right) \subset \mathbf{A}_{0}^{k} .
$$

In particular, this gives rise to a map

$$
d_{\varepsilon}: \mathbf{A}_{0} / \mathbf{A}_{0}^{2} \rightarrow \mathbf{A}_{0} / \mathbf{A}_{0}^{2},
$$

for which $d_{\varepsilon}^{2}=0$. This is a "linearization" of the differential $d$.

For a crossing $\mathbf{x}$, we set $\mathbf{x}^{\varepsilon}=\mathbf{x}+\varepsilon(\mathbf{x}) \in \mathbf{A}$. Obviously, $\mathbf{x}^{\varepsilon} \in \operatorname{Ker} \varepsilon=\mathbf{A}_{0}$. Moreover, the correspondence $\mathbf{x} \mapsto \mathbf{x}^{\varepsilon}$ defines an isomorphism between the vector space $\mathcal{A}$ spanned by the crossings of the $x y$-diagram and $\mathbf{A}_{0} / \mathbf{A}_{0}^{2}$. Hence, $d_{\varepsilon}$ can be regarded as a differential in $\mathcal{A}$. If the augmentation $\varepsilon$ is graded, then $d_{\varepsilon}$ is homogeneous of degree -1 .

In practice, $d_{\varepsilon}: \mathcal{A} \rightarrow \mathcal{A}$ is calculated as follows. We take the formula for $d(\mathbf{a})$ and replace all the crossings $\mathbf{x}$ in the polynomial on the right hand side of the formula by $\mathbf{x}^{\varepsilon}$. The resulting polynomial will have zero constant term, since $\varepsilon \circ d=0$. Then, we erase all monomials of degree $>1$; the result will be a linear combination of crossings, which is $d_{\varepsilon}(\mathbf{a})$.

Let $\mathcal{H}_{\varepsilon}=\operatorname{Ker} d_{\varepsilon} / \operatorname{Im} d_{\varepsilon}$; this is a finite dimensional vector space, which is

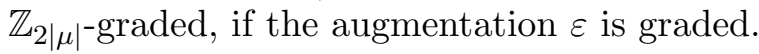

Let $I$ be the set of nonnegative integers $\operatorname{dim} \mathcal{H}_{\varepsilon}$ computed for all possible augmentations $\varepsilon: \mathbf{A} \rightarrow \mathbb{Z}_{2}$, and let $I_{\mathrm{gr}}$ be the set of all Poincaré polynomials of $\mathcal{H}_{\varepsilon}$ computed for all possible graded augmentations $\varepsilon: \mathbf{A} \rightarrow \mathbb{Z}_{2}$.

Theorem $3.9([\mathbf{C}])$. I and $I_{\mathrm{gr}}$ are Legendrian isotopy invariants.

\section{Computing Chekanov-Eliashberg invariants for Eliashberg knots.}

We will show how the invariant $I_{\text {gr }}$ can be used to prove the result stated in the introduction.

Theorem 4.1. The Legendrian knots $E(k, l)$ and $E\left(k^{\prime}, l^{\prime}\right)$ are Legendrian isotopic if and only if $k=k^{\prime}, l=l^{\prime}$ or $k=l^{\prime}, l=k^{\prime}$.

Our proof relies on a partial computation of $I_{\mathrm{gr}}(E(k, l))$. In order to make this computation, we translate the $x z$-diagram in Figure 1 into an $x y$-diagram. A crossing in the $x y$-projection corresponds to a pair of points in the $x z$-projection, $\left(x_{1}, z_{1}\right)$ and $\left(x_{2}, z_{2}\right)$, such that $x_{1}=x_{2}$ and slope at $x_{1}=$ slope at $x_{2}$. We will call such a pair of points a gate. Since we will not allow multiple crossings, we must shift the gates off each other by modifying the $x z$-diagram in Figure 1 to produce the Legendrian isotopic one shown in Figure 17. The gates are labeled in this picture as $\mathbf{a}_{1}, \ldots, \mathbf{a}_{k}, \mathbf{b}_{1}, \ldots, \mathbf{b}_{l}, \mathbf{c}_{1}, \mathbf{c}_{2}, \mathbf{d}_{1}, \mathbf{d}_{2}, \mathbf{d}_{3}, \mathbf{d}_{4}, \mathbf{e}_{1}, \mathbf{e}_{2}, \mathbf{e}_{3}, \mathbf{e}_{4}, \mathbf{f}, \mathbf{p}_{1}, \ldots, \mathbf{p}_{k}, \mathbf{q}_{1}, \ldots$ $\mathbf{q}_{l}$. 


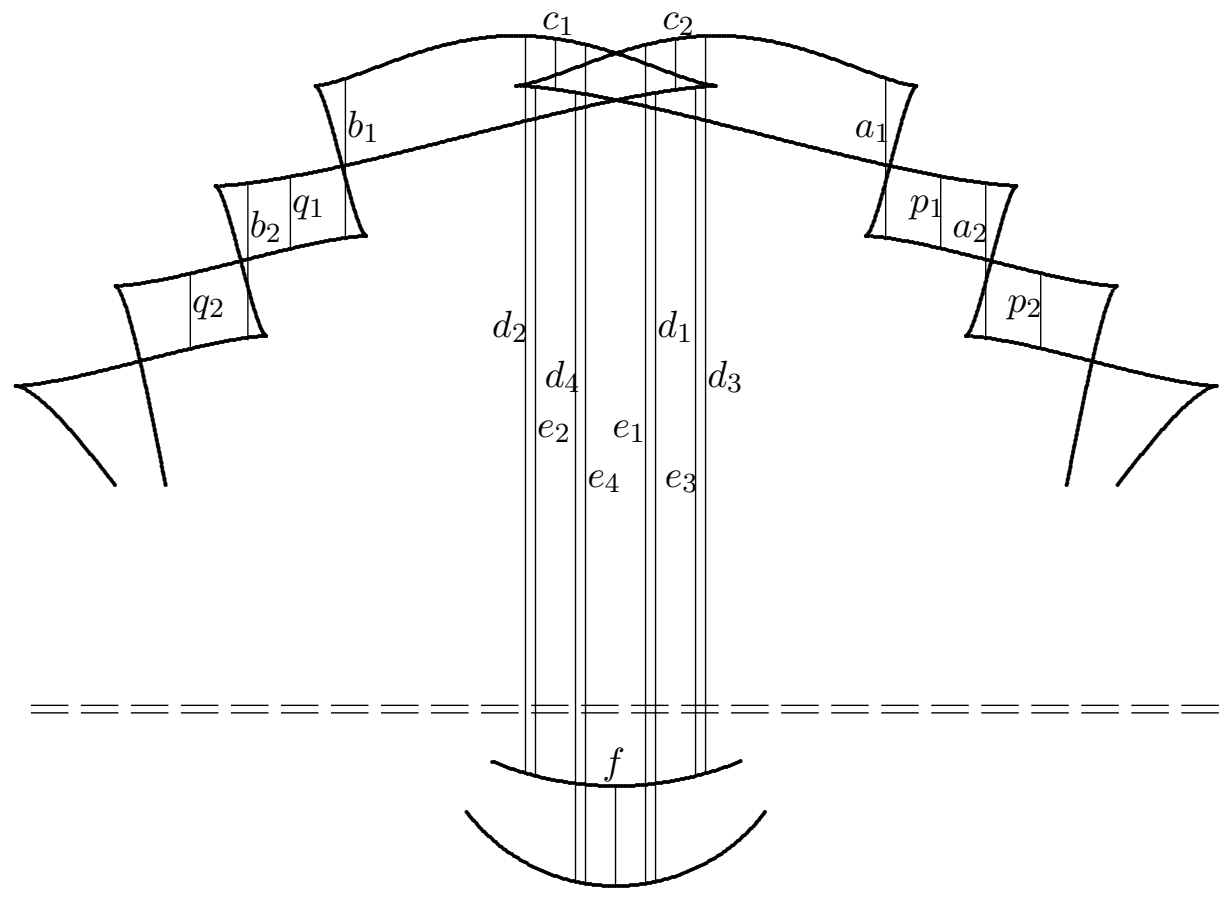

Figure 17.

The $x y$-projection of $E(k, l)$ is presented in Figure 18 .

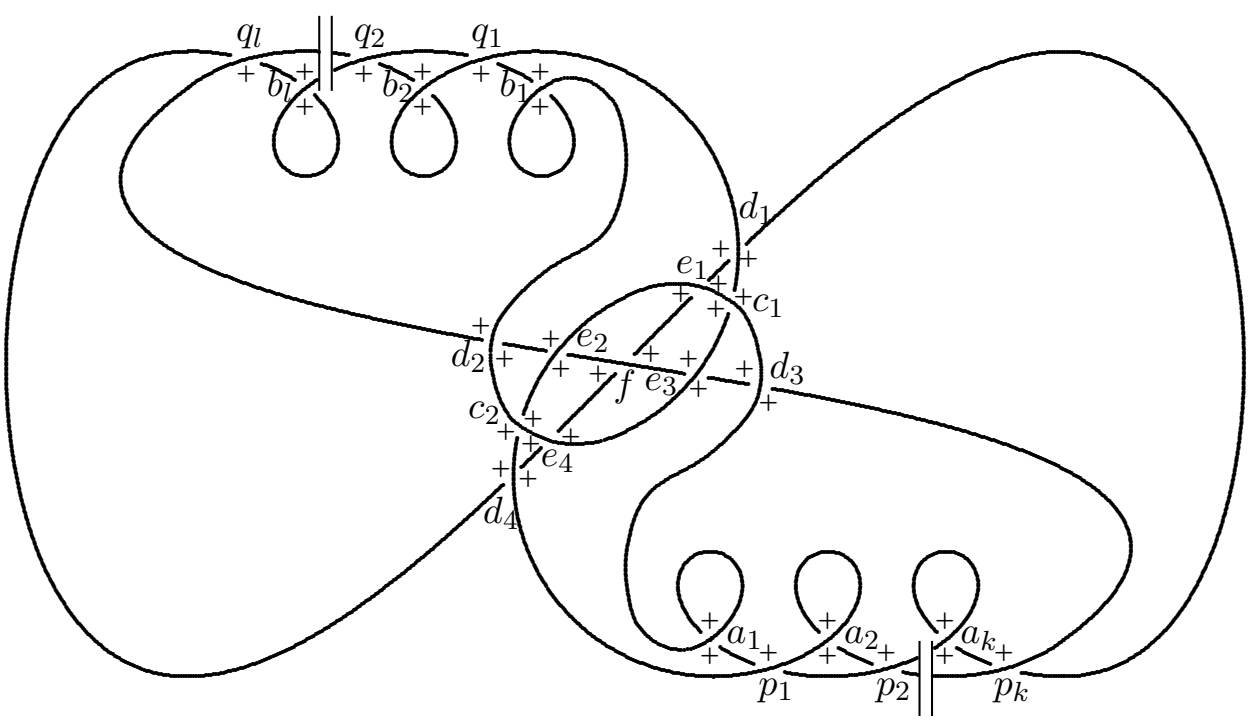

Figure 18. 
We recall that the Chekanov-Eliashberg algebra, corresponding to a generic $x y$-diagram of a Legendrian knot, is generated by the crossings of this diagram. A calculation based on the diagram in Figure 18 yields the results below.

Proposition 4.2. The grading in the Chekanov-Eliashberg complex is as follows:

$$
\begin{gathered}
\operatorname{deg}\left(\mathbf{a}_{i}\right)=1, \operatorname{deg}\left(\mathbf{b}_{i}\right)=1, \operatorname{deg}(\mathbf{f})=1 ; \\
\operatorname{deg}\left(\mathbf{p}_{i}\right)=0, \operatorname{deg}\left(\mathbf{q}_{i}\right)=0 ; \\
\operatorname{deg}\left(\mathbf{c}_{1}\right)=k-l, \operatorname{deg}\left(\mathbf{c}_{2}\right)=l-k ; \\
\operatorname{deg}\left(\mathbf{d}_{1}\right)=l+1, \operatorname{deg}\left(\mathbf{d}_{2}\right)=l+1, \operatorname{deg}\left(\mathbf{d}_{3}\right)=k+1, \operatorname{deg}\left(\mathbf{d}_{4}\right)=k+1 ; \\
\operatorname{deg}\left(\mathbf{e}_{1}\right)=k+2, \operatorname{deg}\left(\mathbf{e}_{2}\right)=k, \operatorname{deg}\left(\mathbf{e}_{3}\right)=l, \operatorname{deg}\left(\mathbf{e}_{4}\right)=l+2 .
\end{gathered}
$$

Proposition 4.3. The following formulas hold for the differential in the Chekanov-Eliashberg complex, corresponding to our diagram.

$$
\begin{aligned}
& d\left(\mathbf{a}_{1}\right)=\mathbf{1}+p_{1}+c_{1} c_{2} p_{1}, \quad d\left(\mathbf{b}_{1}\right)=\mathbf{1}+q_{1}+c_{2} c_{1} q_{1}, \\
& d\left(\mathbf{a}_{2}\right)=\mathbf{1}+p_{1} p_{2}, \quad d\left(\mathbf{b}_{2}\right)=\mathbf{1}+q_{1} q_{2},
\end{aligned}
$$

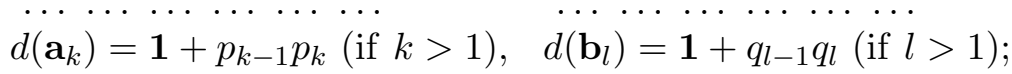

$$
\begin{aligned}
& d\left(\mathbf{c}_{1}\right)=0, d\left(\mathbf{c}_{2}\right)=0, \quad d\left(\mathbf{p}_{i}\right)=0, d\left(\mathbf{q}_{i}\right)=0 ; \\
& d(\mathbf{f})=\mathbf{p}_{k}+q_{l} .
\end{aligned}
$$

Remark. The formulas for $d\left(\mathbf{d}_{i}\right), d\left(\mathbf{e}_{i}\right)$ are much more complicated; see $[\mathbf{M}]$ for details.

Next, we consider augmentations of the Chekanov-Eliashberg complex.

Proposition 4.4. For any $k, l$ the Chekanov-Eliashberg algebra of our diagram has at least one graded augmentation.

Proof. We set $\varepsilon\left(\mathbf{p}_{i}\right)=\varepsilon\left(\mathbf{q}_{i}\right)=1, \varepsilon(\mathbf{x})=0$ for $\mathbf{x} \neq \mathbf{p}_{i}, \mathbf{q}_{i}$ and extend $\varepsilon$ to a ring homomorphism of the Chekanov-Eliashberg algebra to $\mathbb{Z}_{2}$. It follows from Propositions 4.2 and 4.3 , that if $\operatorname{deg}(\mathbf{x}) \neq 0$, then $\varepsilon(d(\mathbf{x}))=0$. Hence, $\varepsilon$ is a graded augmentation.

Remark. If $k \neq l$, then the graded augmentation above is unique; if $k=l$, then there are two more graded augmentations.

Proposition 4.5. If $k \neq l$, then any polynomial in $I_{\mathrm{gr}} E(k, l)$ has precisely one term of negative degree, namely $t^{-|k-l|}$; if $k=l$, then no polynomial in $I_{\mathrm{gr}} E(k, l)$ has a term of negative degree. 
Proof. According to Proposition 4.2, all crossings of our diagram, with the possible exception of $\mathbf{c}_{1}, \mathbf{c}_{2}$ have nonnegative degrees. If $k \neq l$, then one of the crossings $\mathbf{c}_{1}, \mathbf{c}_{2}$ has negative degree, namely, $-|k-l|$; if $k=l$, then all the degrees are nonnegative. Since $d\left(\mathbf{c}_{i}\right)=0$, this proves the proposition.

Proof of Theorem 4.1. Unless $k+l=k^{\prime}+l^{\prime}, E(k, l)$ and $E\left(k^{\prime}, l^{\prime}\right)$ are not even topologically isotopic, and hence not Legendrian isotopic. If $k=l^{\prime}$ and $l=$ $k^{\prime}$, then a rigid rotation of $E(k, l)$ produces $E\left(k^{\prime}, l^{\prime}\right)$. Finally, Proposition 4.5 shows that if $|k-l| \neq\left|k^{\prime}-l^{\prime}\right|$, then $I_{\mathrm{gr}}(E(k, l)) \neq I_{\mathrm{gr}}\left(E\left(k^{\prime}, l^{\prime}\right)\right)$. This completes our proof.

\section{References}

[B] D. Bennequin, Entrelacements et equations de Pfaff, Asterisque, 107-108 (1983), 87-161, MR 86e:58070, Zbl 573.58022.

[C] Yu.V. Chekanov, Differential algebras of Legendrian links, preprint, 1997; revised version, 1999.

[E1] Ya. Eliashberg, Talks at seminars and conferences, private communications.

[E2] _ Invariants in contact topology, Proc. Int. Congr. Mat., II; Doc. Math. 1998, Extra Vol. II, 327-338 (electronic), MR 2000a:57068, Zbl 913.53010.

[FT] D. Fuchs and S. Tabachnikov, Invariants of Legendrian and contact knots in the standard contact space, Topology, 36(5) (1997), 1025-1053, MR 99a:57006, Zbl 904.57006.

[M] M. Meyer, Computation of Chekanov-Eliashberg Invariants for Legendrian Knots, Dissertation, University of California, Davis CA, 1999.

Received March 17, 2000 and revised July 8, 2000.

University of CALifornia, Davis

DAVIS, CA 95616

E-mail address: fuchs@math.ucdavis.edu 